\title{
Comparative study of extreme ultraviolet absorber materials using lensless actinic imaging
}

\author{
Sara Fernandez, ${ }^{\mathrm{a}, *}$ Dimitrios Kazazis, ${ }^{\text {a }}$ Rajeev Rajendran, ${ }^{\mathrm{a}}$ lacopo Mochi, ${ }^{a}$ Patrick Helfenstein, ${ }^{\mathrm{a}}$ \\ Shusuke Yoshitake, ${ }^{b}$ and Yasin Ekinci ${ }^{\mathrm{a}}$ \\ apaul Scherrer Institute, Villigen PSI, Villigen, Switzerland \\ bNuFlare Technology, Inc., Isogo-ku, Yokohama, Japan
}

\begin{abstract}
Background: One of the challenges for extreme ultraviolet (EUV) lithography is the mitigation of mask threedimensional effects arising from the oblique incident angle and the mask topography. As the scanners' numerical aperture and the pattern aspect ratio increase, these effects become more prominent. A potential solution to reduce them consists in replacing the current TaBN absorber for a higher-k material.

Aim: We demonstrate the potential of a mask inspection platform to evaluate the impact of different absorber materials on actinic defect inspection.

Approach: We evaluate the performance of a reflective-mode EUV mask scanning microscope (RESCAN), our actinic lensless inspection tool, with three different absorber materials (hydrogen silsesquioxane, TaBN, and Ni). We study the effect of these materials on the image formation and compare the defect maps.

Results: The Ni absorber mask exhibits a better contrast compared to the TaBN one, even though the thickness of the layers differs only by $10 \mathrm{~nm}$. Programmed defects are localized and detected with a high signal-to-noise ratio (SNR).

Conclusions: The gain in contrast for the Ni absorber being significant, the SNR is higher for a smaller defect in a TaBN absorber photomask. RESCAN allows the evaluation of the performance of absorber materials in defectivity and image formation on small samples.
\end{abstract}

(C) 2019 Society of Photo-Optical Instrumentation Engineers (SPIE) [DOI: 10.1117/1.JMM.18.1.013506]

Keywords: extreme ultraviolet lithography; extreme ultraviolet absorber; photomask; actinic inspection; coherent diffraction imaging.

Paper 18125 received Oct. 9, 2018; accepted for publication Feb. 28, 2019; published online Mar. 18, 2019.

\section{Introduction}

Extreme ultraviolet (EUV) photomask technology, including fabrication, characterization, and inspection, is of key importance to make EUV lithography a cost-effective technology for high-volume semiconductor manufacturing. In this context, one of the core challenges is the mitigation of mask three-dimensional (3-D) effects, such as pitch-dependent focus shift, induced pattern asymmetries, and image contrast loss. Indeed, mask topography effects become more important as the mask feature sizes decrease, so that their origin and influence in aerial images must be carefully investigated and mitigated. ${ }^{1,2}$

Among the strategies to reduce the impact of the mask 3-D effects, reducing the thickness of the absorber layer, i.e., changing the material of the absorber stack with a high-k absorber while keeping the optical contrast similar, has been widely studied with numerical simulations. ${ }^{1-5}$ Nevertheless, the fabrication of such samples remains challenging. ${ }^{3}$ A direct experimental comparison between different absorbers and the impact of the chosen materials on aerial image formation is critical. By using actinic inspection, the amount or/and the kind of defects found within a mask may help in discarding or promoting a certain material. Furthermore, the use of different absorber materials allows the possibility of

*Address all correspondence to Sara Fernandez, E-mail: sara.fernandez@psi ch evaluating the resolution of the inspection tool itself as a function of the contrast for a given thickness of the chosen absorber stack.

In this paper, the imaging performance of reflective-mode EUV mask scanning microscope (RESCAN) on masks with three different absorber materials is presented. RESCAN is used for EUV mask inspection and it offers high resolution by employing coherent diffraction imaging (CDI) methods to retrieve both the magnitude and the phase of the sample, thereby allowing the localization of both amplitude and phase defects with a very high sensitivity. ${ }^{6-10}$

In previous works, we have demonstrated the capability of RESCAN to detect programmed defects as small as $50 \mathrm{~nm}$ on random patterns fabricated in-house. ${ }^{9}$ In our studies, the absorber consisted of a hydrogen silsesquioxane (HSQ) layer with 140-nm thickness. Here, we use an analogous sample to be compared with a state-of-the-art EUV mask, which is a 70-nm-thick TaBN absorber, and a 60-nm-thick Ni layer, where the latter is among the preferred materials to optimize the absorber physical properties. ${ }^{3,4,11}$ In Sec. 2, we explain the choice of these absorber materials and their optical properties and describe the samples investigated during the experiments presented here (Sec. 2.1). Then, the instrumental setup and technique employed in RESCAN are described (Sec. 2.2). The results in Sec. 3 show the comparative

$1932-5150 / 2019 / \$ 25.00$ @ 2019 SPIE 


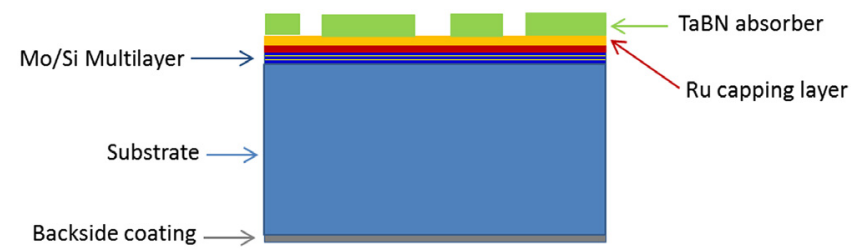

Fig. 1 Schematic representation of the Ta-based EUV photomask measured in this work, which is the state-of-the-art mask in EUV photomask technology.

analysis of the imaging properties of different samples as well as the die-to-die inspection results.

\section{EUV Mask Absorber Materials and Inspection Evaluation}

EUV mask stacks consist of a MoSi multilayer (ML), generally composed of 40 to 50 bilayers, a Ru-based capping layer on top, and an absorber structure, as sketched in Fig. 1. The pattern is defined by selectively etching the absorber material on top of the ML mirror and the interaction of the incident light with the mirror itself. The contrast in the image is provided by the intensity difference between the light reflected by the mirror and the light transmitted through the absorber layer. Ta-based materials, e.g., TaN and TaBN, provide high absorption and are the state-of-the-art materials in EUV lithography $(\lambda=13.5 \mathrm{~nm})$. Nevertheless, a thickness of $70 \mathrm{~nm}$ is needed to ensure acceptable absorption, ${ }^{3}$ which induces the so-called mask 3-D effects in the resulting projected images at nonzero angle of incidence. Moreover, these effects are likely to become more prominent as the pattern critical dimension $(\mathrm{CD})$ decreases. Different routes to mitigate these effects are currently being investigated, e.g., completely removing the absorber layer and etching the ML, ${ }^{12,13}$ changing the EUV mask structure, ${ }^{14}$ introducing phase shifting masks ${ }^{15}$ or considering other absorber materials that have stronger absorption, i.e., higher k-values than $\mathrm{Ta}$, such as Ni or Co. ${ }^{1,4}$ Here, we focus on the choice of alternative materials and evaluate the imaging performances after the reduction of the absorber thickness.

\subsection{Samples Description}

When choosing the most suitable absorber material for EUV masks, several factors play a role, such as their optical properties at EUV wavelength, the feasibility of patterning with standard nanofabrication methods and the line-edge roughness (LER) of the resulting absorber pattern. The contrast between the absorbed and the reflected light must be enhanced. As previously mentioned, the absorber thickness has to be reduced as much as possible to minimize the proximity effects due to the oblique incident illumination. Hence, a high absorption coefficient $(k)$ and a refractive index $(n)$ as close to 1 as possible are desirable. The optical properties of the absorbers evaluated in this study are displayed in Table 1, in which we have included the thickness of the layers forming our samples. Note that, in the case of $\mathrm{Ni}$, the optimal thickness depends on the absorber coefficient and could be optimized. ${ }^{4,16}$ However, obtaining thin, smooth, and defect-free Ni layers on ML stacks without damaging the ML is extremely difficult. ${ }^{17,18}$ Also, in the case of HSQ, the index of refraction depends on the development parameters after electron beam exposure, as density and composition may
Table 1 Optical properties and thickness of the materials chosen as absorbers in this study. ${ }^{6,15}$

\begin{tabular}{lccc}
\hline & HSQ & TaBN & Ni \\
\hline Index of refraction $(n)$ & $0.9781-0.9843$ & 0.925 & 0.948 \\
Absorber coefficient $(k)$ & $0.005-0.008$ & 0.044 & 0.073 \\
Thickness (nm) & 140 & 70 & 60 \\
Attenuation length $(\mathrm{nm})$ & $217-135$ & 22.7 & 14.7 \\
\hline \hline
\end{tabular}

vary. ${ }^{19}$ The given range corresponds to a density between 1.57 and $2.17 \mathrm{~g} / \mathrm{cm}^{3} .^{20}$

To compare the influence of different absorber materials on the CDI reconstructions, we used a TaBN EUV mask (see Fig. 1) (manufactured by Nuflare Technology Inc. and Dai Nippon Printing Co., Ltd.), representing the state-of-the-art absorber stack. We manufactured EUV masks with HSQ and $\mathrm{Ni}$ absorber layers in-house. Both layers were deposited on a silicon wafer with a MoSi ML designed for high reflectivity at 6-deg angle of incidence and 13.5-nm wavelength. We chose HSQ due to its patterning simplicity. HSQ is a highresolution, negative-tone e-beam resist, yielding $\mathrm{SiO}_{2}$ patterns subsequent to exposure and development. For the fabrication of the Ni sample, we carried out extensive process optimization to obtain high-quality patterns despite the fact that Ni was prone to produce high LER due to its crystallinity. We noted that the present thicknesses of the HSQ and Ni absorbers were not optimized. We used LER as a metric to compare the quality of the patterns in the three absorber materials. To evaluate the LER we collected SEM images of a lines-and-spaces pattern following the imec protocol outlined in Ref. 21. We measured the profiles of six lines with a CD of $200 \mathrm{~nm}$ and a length of $3 \mu \mathrm{m}$; we calculated the unbiased average power spectral density and we integrated it to get the LER. We measured LER values of 5.37, 5.72, and 6.37 for the HSQ, TaBN, and Ni masks, respectively. The LER of the Ni mask might be improved using a nickel alloy to reduce its crystallinity. ${ }^{21}$

The layout of the EUV masks consists of a nonperiodic pattern with horizontal power rails and extends over an area of $15 \times 16 \mu \mathrm{m}^{2}$, as shown in Fig. 2(a). SEM images of the three samples are displayed in Figs. 2(b)-2(d). The measured $\mathrm{CD}$ is $114.4 \mathrm{~nm}$ for the TaBN sample, and $218 \mathrm{~nm}$ for the samples that are fabricated in-house (HSQ and Ni). Every sample contains a single programmed defect, consisting of an extrusion in the center of the pattern, as will be further explained in Sec. 3.2.

\subsection{Methods}

To investigate the masks described in Sec. 2.1., we employed a scanning coherent diffraction method known as ptychography. The three photomasks were inspected using the RESCAN tool installed at the XIL-II beamline at the Swiss light source (SLS). ${ }^{22}$ The incoming coherent beam $(\lambda=$ $13.5 \mathrm{~nm}$ ) was focused using a condenser and a folding mirror with an incident angle of $6 \mathrm{deg}$, as depicted in Fig. 3. Detailed descriptions of the beamline and setup can be found elsewhere. ${ }^{9,22}$ 


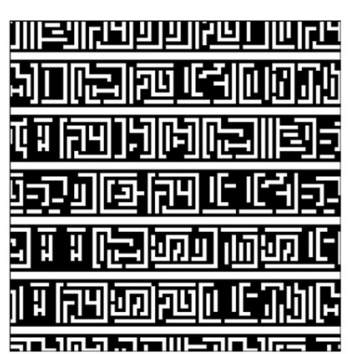

(a)

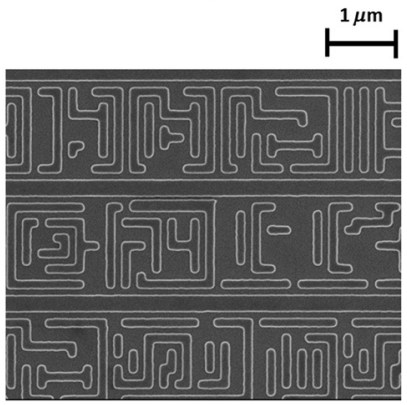

(c)

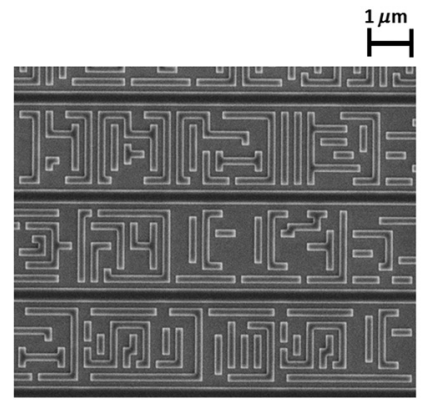

(b)

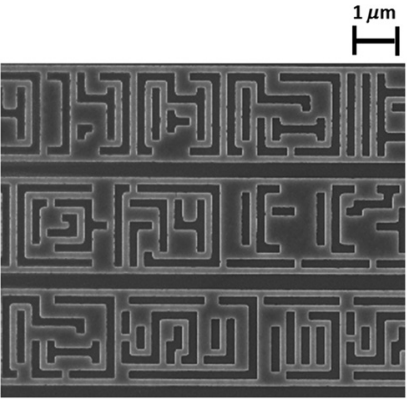

(d)
Fig. 2 (a) Layout of the unit cell of the random pattern $\left(15 \times 16 \mu \mathrm{m}^{2}\right)$ (b) and high resolution SEM images on the center of the fields of the HSQ, (c) TaBN, and (d) Ni absorber samples, respectively.

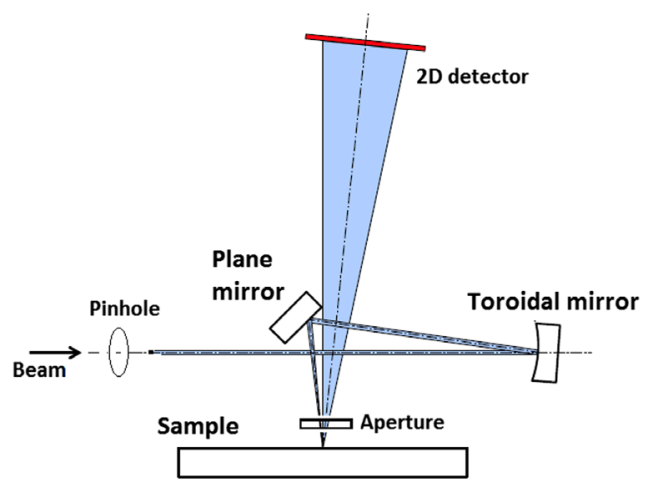

Fig. 3 RESCAN experimental setup at the XIL-II beamline. The aperture on top of the sample is used for the HSQ sample to avoid scattering from the blank ML outside the patterned field.

The masks were scanned with a high-precision piezoelectric positioning stage (accuracy of $\pm 3 \mathrm{~nm}$ ), whereas the scattered intensity from the reflective mask was collected by a two-dimensional detector with $2048 \times 2048$ pixels of $13.5-\mu \mathrm{m}$ size. Given the spot size (FWHM) of the incident illumination of about $3 \mu \mathrm{m}$ on the sample plane, a step size of $1 \mu \mathrm{m}$ was chosen to guarantee a minimum overlap of $60 \%$.

To record the higher spatial frequencies, longer exposure times are warranted. However, this leads to saturation at lower frequencies prevalent in the CCD camera. To overcome this, several diffraction patterns with different exposure times at every scan position are recorded, thereby ensuring the coverage of both low and high spatial frequencies without saturation at a high signal-to-noise ratio (SNR). The exposure times range from $100 \mathrm{~ms}$ to $60 \mathrm{~s}$, and the images are stitched together as explained in Refs. 23-25. The diffraction data sets are then processed with the iterative algorithms ${ }^{23,24}$ to recover simultaneously both the amplitude and the phase maps of the masks, as well as the complex illumination probe field.

\section{Experimental Results}

\subsection{Results: Comparison between Absorbers}

An area of $15 \times 15 \mu \mathrm{m}^{2}$ of each sample was scanned. The diffraction data sets were used as input of the difference map engine ${ }^{26,27}$ to reconstruct the amplitude and the phase of the imaged photomasks. This algorithm uses the redundancy on the data, i.e., the common information in two diffraction patterns at consecutive positions of the scan to retrieve the experimentally missed phase. ${ }^{28}$ Two mixedstates in the description of the illumination probe were implemented in order to compensate partial coherent or sample vibrations during the measurements. ${ }^{29}$ The algorithm was run for about 600 iterations until changes to the reconstructed images between iterations became insignificant.

Figure 4 shows the reconstructed actinic images (amplitude maps) of about $10 \times 10 \mu \mathrm{m}^{2}$ area of the masks made by the HSQ and $\mathrm{Ni}$ absorbers and the equivalent one of about $6 \times 6 \mu \mathrm{m}^{2}$ of the TaBN absorber (smaller CD). The achieved pixel resolution is $34 \mathrm{~nm}$. The reconstructed amplitude images reproduce in both cases the mask design with high fidelity. To compare the contrast of the independent reconstructions, we define the contrast $C$ as

$C=\frac{\overline{I_{M}}-\overline{I_{m}}}{\overline{I_{M}}+\overline{I_{m}}}$

where $\overline{I_{M}}$ and $\overline{I_{m}}$ denote the average intensities of the bright and dark regions, respectively. Using this definition, we find relative values of $51 \%, 59 \%$, and $67 \%$ for the HSQ, TaBN, and $\mathrm{Ni}$ masks, respectively. As expected, the retrieved images show an enhancement of contrast in the case of the thinnest absorber, i.e., the $\mathrm{Ni}$ one. The $8 \%$ increase of contrast of the $\mathrm{Ni}$ mask with respect to the TaBN mask is remarkable for the 10-nm thinner absorber, owing to the lower absorber coefficient of $\mathrm{Ni}$ (see Table 1). Note that the contrast values are relative. Quantitative optical analysis of the reconstructions is limited by the flare and random noise added to the background in the ptychographic retrieving process.

The retrieved phase maps are shown in Fig. 5. The phase contrast measured from the reconstructed images yields phase differences of $\Delta \phi_{\mathrm{HSQ}}=2.72 \pm 0.2 \mathrm{rad}, \Delta \phi_{\mathrm{TaBN}}=$ $2.19 \pm 0.3 \mathrm{rad}$, and $\Delta \phi_{\mathrm{Ni}}=2.74 \pm 0.5 \mathrm{rad}$, respectively. For absorber materials, the phase change is related to the thickness of the layer as follows: ${ }^{9}$

$\Delta \phi=\frac{4 \pi h}{\lambda}\left(\frac{1}{\cos \theta}-\frac{n^{2}}{\sqrt{n^{2}-\sin ^{2} \theta}}\right)$,

where $h$ is the height of the sample, $\lambda$ is the wavelength, and $\theta_{i}$ is the incident angle. Using the refractive index and the measured thickness values shown in Table 1, we get $\Delta \phi_{\mathrm{TaBN}}=2.258 \mathrm{rad}$ and $\Delta \phi_{\mathrm{Ni}}=2.89$, which are consistent with the measurement obtained with RESCAN. For the HSQ sample, we calculated the maximum and minimum values associated with the two limits of the refractive index: 

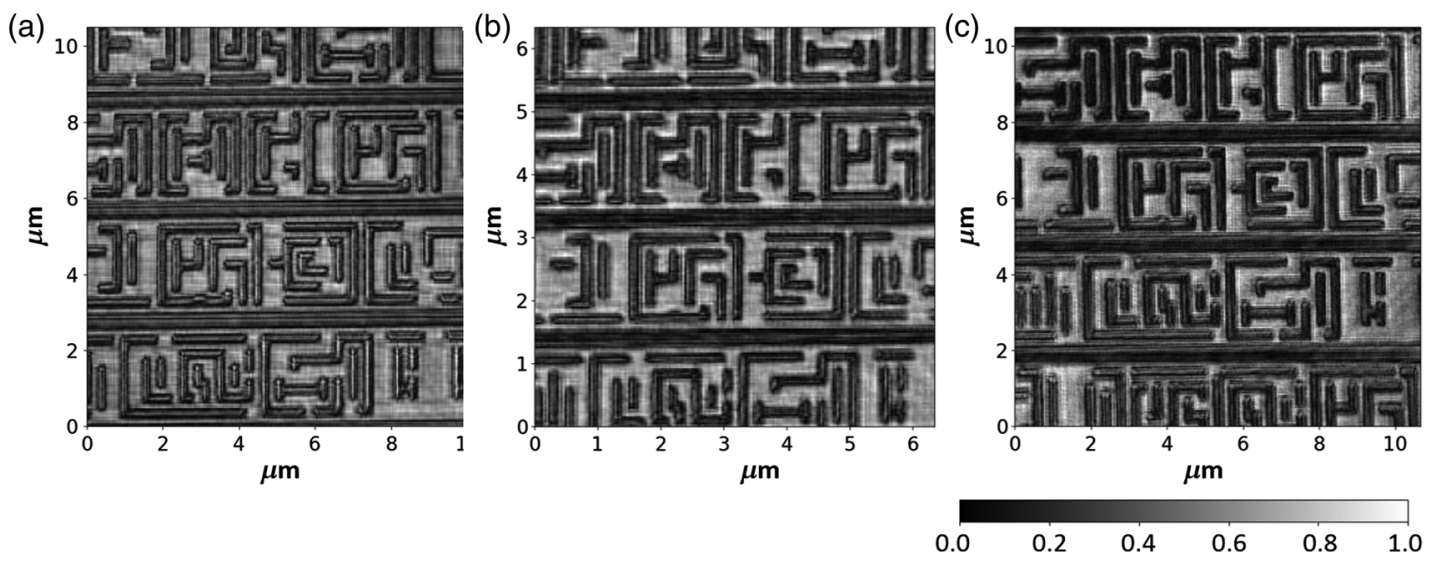

Fig. 4 Zoomed image section of the reconstructed magnitudes of the (a) HSQ, (b) TaBN, and (c) Ni absorber masks. The color bar applies to all images.
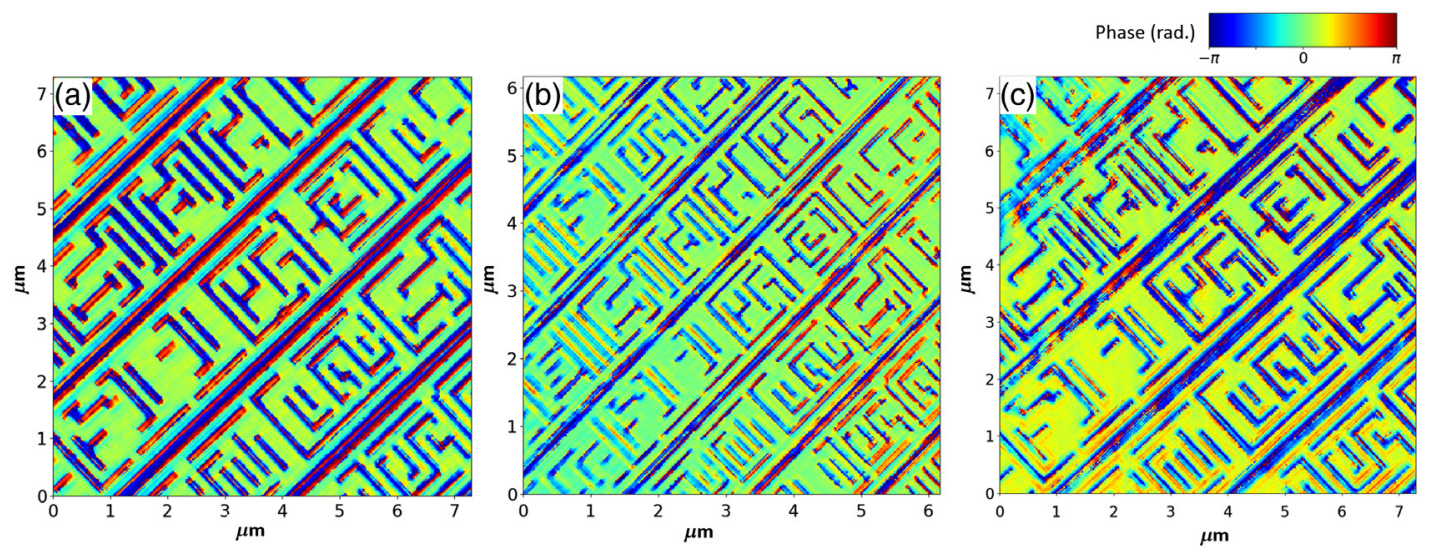

Fig. 5 Zoomed image of the center of the reconstructed phase maps of the (a) HSQ, (b) TaBN, and (c) $\mathrm{Ni}$ absorber masks. The color bar applies to all maps.

$\Delta \phi_{\mathrm{HSQ} \text { min }}=2.04 \mathrm{rad}$ and $\Delta \phi_{\mathrm{HSQ} \max }=3.04 \mathrm{rad}$. The measured phase difference is consistent with these values, but we would need a measurement of the sample refractive index to make an accurate comparison. As expected for the given thicknesses, the phase contrast is highest in the case of $\mathrm{Ni}$.

The present study, yielding high-contrast images, shows the capability of RESCAN to perform EUV actinic mask inspection with very good spatial resolution and sensitivity, independent of the chosen absorber material. Our study shows that a thin absorber improves the magnitude contrast, whereas HSQ gives a strong phase contrast with low amplitude contrast.

\subsection{Actinic Inspection and Defect Sensitivity}

As introduced in Sec. 2.1, programmed defects were printed on every mask. Next to the field depicted in Sec. 2, Fig. 2(a), a second pattern, equal to the previous one but containing a programmed defect, was reproduced. The defect consisted of an extrusion of $64 \times 32 \mathrm{~nm}^{2}$ in the TaBN mask, and $269 \times$ $70 \mathrm{~nm}^{2}$ size in the HSQ and Ni absorber masks due to the different CDs.

A die-to-die inspection can be performed based on the magnitude or phase maps using the reconstructed images. Zooms on the amplitude maps of the reference and defect fields are displayed in Fig. 6, in the first and second columns, respectively. The extrusion is circled in red in the reconstructed amplitude. Every row corresponds to one material: HSQ, TaBN, and Ni from top to bottom.

On the third column of Fig. 6, the difference between the defect and the reference images is displayed for every absorber material. The difference is subtracted after alignment of the features to obtain the defect maps, in which the defect in the TaBN photomask turns out to be resolved best, despite its smaller size in both directions. For a better visualization of the intensity arising from the defect, a thresholded defect map is shown in the fourth column of the same figure.

The defective SNR of these images can be calculated using the definition: ${ }^{9}$

$\mathrm{SNR}=\frac{\overline{I_{D}}-\overline{I_{A}}}{\operatorname{std}(A)}$,

where $\operatorname{std}(A)$ is the standard deviation of the magnitude of the image, and $\left(\overline{I_{D}}\right)$ and $\left(\overline{I_{A}}\right)$ are the average magnitude values calculated on the defect pixels of the image and on the whole image, respectively. The results are summarized in Table 2. The highest SNR is found for TaBN. We note that the reported SNR values are based on the average rather than on the integrated signal of all the defective pixels and are therefore independent of the footprint of the defects. 


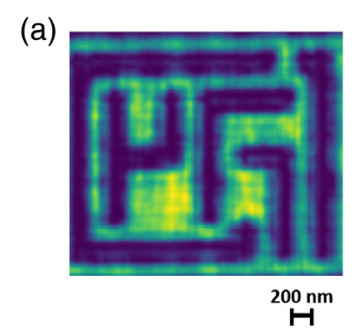

(b)

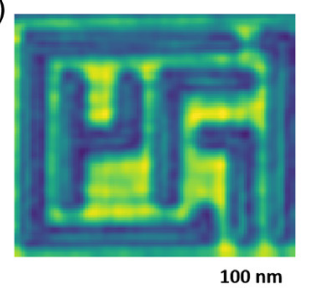

(c)

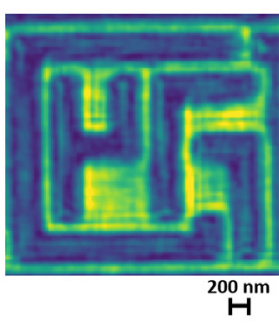

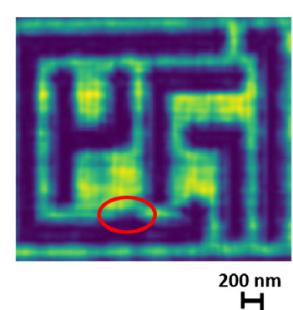

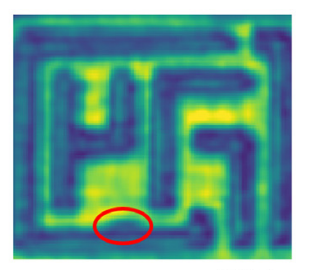

$100 \mathrm{~nm}$
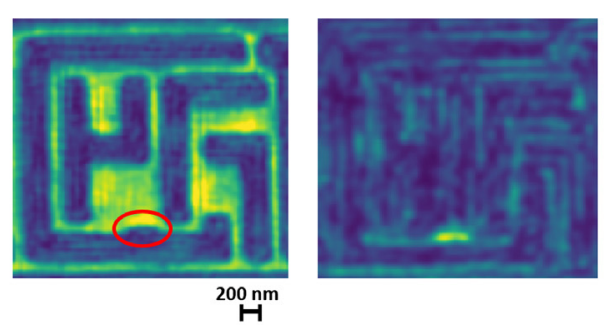
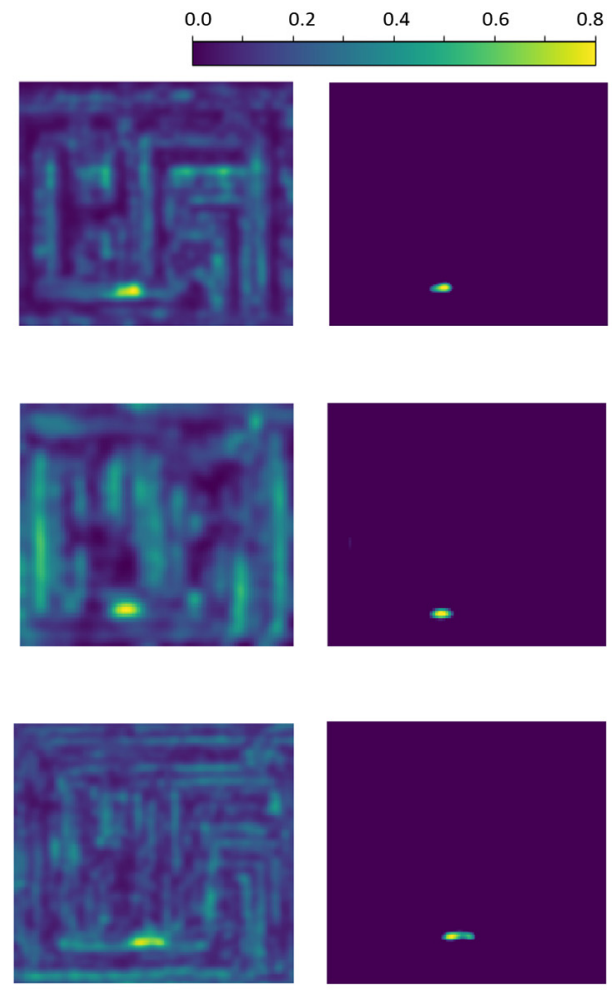

Fig. 6 From left to right: reference and defect structures, defect maps, and thresholded defect map of the samples fabricated with (a) HSQ, (b) TaBN, and (c) Ni as absorber materials.

Table 2 Defect sizes on the measured sample and SNR.

\begin{tabular}{lccc}
\hline & HSQ & TaBN & Ni \\
\hline Defect size $\left(\mathrm{nm}^{2}\right)$ & $269 \times 70$ & $64 \times 35$ & $269 \times 70$ \\
SNR & 6.85 & 7.89 & 7.64 \\
Thickness $(\mathrm{nm})$ & 140 & 70 & 60 \\
\hline \hline
\end{tabular}

\section{Conclusions and Outlook}

Three EUV photomasks with different absorber materials, i.e., HSQ, TaBN, and $\mathrm{Ni}$, were prepared and evaluated from the actinic inspection point of view. Lensless imaging showed a better contrast for the thinnest layer, $\mathrm{Ni}$, followed by TaBN and HSQ. On the other hand, the retrieved phases matched the calculated ones from the height of the absorber stacks.

Programmed defects of sizes ranging from 35- to 70-nm minimum lateral sizes could be located and evaluated in RESCAN through a die-to-die approach. A higher SNR is found for the TaBN absorber, despite the fact that it exhibited a lower contrast than Ni. Further investigations are necessary to generalize this result and make conclusions about the defect detection sensibility as a function of the material. A reduction of the $\mathrm{Ni}$ thickness would be advantageous for the comparison of the materials, as well as the reproduction of the same layout and CDs.

Manufacturability of EUV photomasks with alternative absorber materials remains, nevertheless, a concern and needs further work. In this paper, we have shown that RESCAN is able to perform actinic inspection in any of the proposed materials, achieving high resolution and defect sensitivity and opening routes toward the study of other kinds of defects. Potentially, phase defects and shadowing effects at smaller pitches could be studied by taking advantage of the direct measurement of the phase.

\section{Acknowledgments}

The authors would like to thank Markus Kropf, Michaela Vockenhuber, and Li-Ting Tseng for their excellent support during the experiments and Shingo Yoshikawa, Dai Nippon Printing Co., Ltd., Japan, for providing the TaBN masks. Part of this work has been performed at the SLS, Paul Scherrer Institut, Switzerland, and has been presented at SPIE Advanced Lithography 2018. ${ }^{30}$

\section{References}

1. P. Yan et al., "EUV mask absorber characterization and selection," Proc. SPIE 4066, 116-124 (2000).

2. A. Erdmann, P. Evanschitzky, and T. Fühner, "Mask diffraction analysis and optimization for EUV masks," Proc. SPIE 7271, 72711E (2009).

3. A. Rastegar et al., "Study of alternative capping and absorber layers for extreme ultraviolet (EUV) masks for sub-16nm half-pitch nodes," Proc. SPIE 9048, 90480L (2014).

4. V. Philipsen et al., "Reducing EUV mask 3D effects by alternative metal absorbers," Proc. SPIE 10143, 1014310 (2017).

5. F. Scholze et al., "Update on optical material properties for alternative EUV mask absorber materials," Proc. SPIE 10446, 1044609 (2017).

6. B. Zhang et al., "Quantitative tabletop coherent diffraction imaging microscope for EUV lithography mask inspection," Proc. SPIE 9050, 90501D (2014).

7. P. Helfenstein et al., "Scanning coherent diffractive imaging methods for actinic extreme ultraviolet mask metrology," J. MicroNanolithogr. MEMS, MOEMS 15(3), 034006 (2016). 
8. I. Mochi et al., "RESCAN: an actinic lensless microscope for defect inspection of EUV reticles," J. MicroNanolithogr. MEMS, MOEMS 16(4), 041003 (2017).

9. I. Mochi et al., "Actinic inspection of EUV reticles with arbitrary pattern design," Proc. SPIE 10450, 1045007 (2017).

10. T. Harada et al., "Mask observation results using a coherent extreme ultraviolet scattering microscope at NewSUBARU," J. Vac. Sci. Technol. B: Microelectron. Nanometer Struct. Process. Meas. Phenom. 27(6), 3203-3207 (2009).

11. O. Wood et al., "Alternative materials for high numerical aperture extreme ultraviolet lithography mask stacks," Proc. SPIE 9422 94220I (2015).

12. B. L. Fontaine et al., "Architectural choices for EUV lithography masks: patterned absorbers and patterned reflectors," Proc. SPIE $\mathbf{5 3 7 4}$ 300-311 (2004).

13. L. V. Look et al., "Alternative EUV mask technology to compensate for mask 3D effects," Proc. SPIE 9658, 96580I (2015).

14. Y. Deng et al., "Rigorous EM simulation of the influence of the structure of mask patterns on EUVL imaging," Proc. SPIE 5037, 302-314 (2003).

15. B. L. Fontaine et al., "Demonstration of phase-shift masks for extremeultraviolet lithography," Proc. SPIE 6151, 61510A (2006).

16. T. Kamo et al. "Effects of mask absorber thickness on printability in EUV lithography with high resolution resist," Proc. SPIE 7028, 70281R (2008).

17. D. Hay et al., "Thin absorber extreme ultraviolet photomask based on Ni-TaN nanocomposite material," Opt. Lett. 41(16), 3791-3794 (2016).

18. C. M. Gonzalez et al., "Evaluation of mask repair strategies via focused electron, helium and neon beam induced processing for EUV applications," Proc. SPIE 9048, 90480M (2014).

19. B. L. Henke, E. M. Gullikson, and J. C. Davis, "X-ray interactions: photoabsorption, scattering, transmission, and reflection at $\mathrm{E}=50-30,000 \mathrm{eV}$, $\mathrm{Z}=1-92, "$ At. Data Nucl. Data Tables 54(2), 181-342 (1993).
20. H.-J. Lee et al., "Structural comparison of hydrogen silsesquioxane based porous low-k thin films prepared with varying process conditions," Chem. Mater.-Chem Mater 14, 1845-1852 (2002).

21. V. Philipsen et al., "Single element and metal alloy novel EUV mask absorbers for improved imaging," Proc. SPIE 10450, 104500G (2017).

22. R. Rajendran et al., "Towards a stand-alone high-throughput EUV actinic photomask inspection tool: RESCAN," Proc. SPIE 10145, $101450 \mathrm{~N}(2017)$

23. N. Mojarad, J. Gobrecht, and Y. Ekinci, "Interference lithography at EUV and soft X-ray wavelengths: principles, methods, and applications," Microelectron. Eng. 143, 55-63 (2015).

24. P. Helfenstein et al., "Beam drift and partial probe coherence effects in EUV reflective-mode coherent diffractive imaging," Opt. Express 26(9), 12242-12256 (2018).

25. D. G. Woo et al., "Coherent scattering microscopy as an effective inspection tool for analyzing performance of phase shift mask," Opt. Express 24(11), 12055-12062 (2016).

26. P. Thibault et al., "Probe retrieval in ptychographic coherent diffractive imaging," Ultramicroscopy 109(4), 338-343 (2009).

27. A. M. Maiden and J. M. Rodenburg, "An improved ptychographical phase retrieval algorithm for diffractive imaging," Ultramicroscopy 109(10), 1256-1262 (2009).

28. D. Sayre, "Some implications of a theorem due to Shannon," Acta Crystallogr. 5(6), 843-843 (1952)

29. P Thibault and A. Menzel, "Reconstructing state mixtures from diffraction measurements," Nature 494(7435), 68-71 (2013).

30. S. Fernandez et al., "A comparative study of EUV absorber materials using lensless actinic imaging of EUV photomasks," Proc. SPIE 10583, 105831H (2018).

Biographies of the authors are not available. 